\title{
Parameter Management in Configuration for the Design of Products Families
}

\author{
Coraline Deffrenne, Mathieu Bettwy, Aurélie Robert, \\ Karine Deschinkel, and Samuel Gomes \\ IRTES-M3M, France \\ \{Coraline.DEFFRENNE, Mathieu.BETTWY, Aurelie.ROBERT, Samuel.GOMES\}@utbm.fr
}

\begin{abstract}
This paper presents a design approach shows the usefulness of the software in the management of parameters in the design of products. Indeed, until now, the definition of this type of interface depends only on knowledge of the designers. It was therefore difficult to group settings to facilitate modular design. To highlight this, the methodology presented in this paper is to describe a journey through various design software to reduce the designers' work. Rules that the designer has put in place during the design of the product, called "design rules" also define the interface. This has the advantage to guide the designer in his work and to allow a rapid evolution of the interface in the case of product development. The objective is to reduce the time of the routine engineering to devote more time to innovation. At the same time, this method allows you to define assembly sequences based on parameters needed to design the product. Some calculations to be carried out before others because the calculated parameters are necessary in one to another. The definition of an ordered graph allows to directly obtain valid assembly sequences necessary for design while offering designers a thread.
\end{abstract}

Keywords: Product design, specific parameters, functional parameters, optimized design sequence, modularity, product range.

\section{Introduction}

To design a product, specifications should be established. To obtain them, the needs, the environment and the functioning are analyzed. This system analysis results in a list of components, parameters, functions and rules, which are sometimes a myriad. This number of criteria, which can be huge, to be taken in consideration requires a lot of work. To spare this time, the specifications can be simplified with the use of some software.

After having analyzed the needs and the environment, the internal functional analysis begins. Depending on the fact that the design of the product is only a redesign or an innovative design, the internal functional analysis can be preceded by the definition of pre-concepts and of the new product structure. During this analysis, the list of components, taking part in the product structure, and of functions are introduced and then organized in a tree structure. To 
each component and to each function, characteristics are associated with values and tolerances : these are respectively the specific parameters and the functional parameters [ROBERT 2011] [1].

This paper introduces the utility of software in the management of parameters during a product design. Because product design is a competitive domain, but also because it spares time for the product designer team, application of software to simplify the work can be an asset. By this way, the definition of the product and its related assembly sequence will be more quickly obtained.

\section{Software}

\subsection{ACSP}

ACSP is a Collaborative Project Monitoring workshop [TOUSSAINT 2010] [2]. It helps designing the project by : the integration of the concept of concurrent engineering, a rapid visualization of the project state and progress, and the project related data management and accumulation.

Its six main features are : user access management (login and password), file management (management of versions), data management (project type, product, process, use), life cycle management and document state, communication management (messages, forums), and accumulation and reuse (project templates, data standards).

\subsection{Kadviser}

Kadviser is a Knowledge Based Engineering (KBE) software [PETIT] [3. A KBE application aims to help mechanical designers by providing them a set of functionalities necessary to use and update the know-how of a company.

This software provides a connection with PLM systems (Product Lifecycle Management), CAD software (CATIA, Nx6, etc.) and Microsoft Office tools (Excel, Word, etc.).

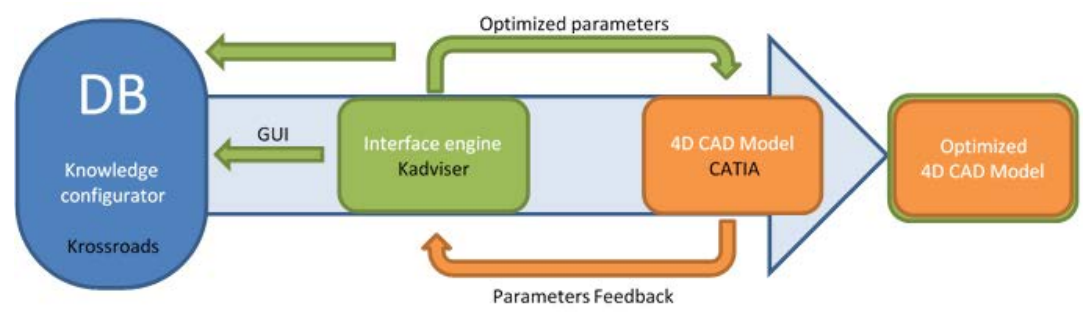

Fig. 1. Kadviser general architecture 
Kadviser is also known for its constraints propagation inference engine. In fact, it does ignore the writing direction of rules. The order has no influence on reasoning. The inference engine applies the principle of reversibility, which means : For the expression "a"+"b"="c", where values "a" and "b" are known, the inference engine may determine the value of "c". If values "a" and "c" are known, it will find "b".

In a design project, it can be coupled with a Collaborative Project Monitoring workshop, such as ACSP.

\subsection{ORASSE Knowledge}

In the product design domain, it exists the FARD (Functional And Robust Design) methodology [ROBERT] [4. Its aim is to design and generate quickly the entire product variety of a sale modular family without forgetting to respect the customer requirements (functional design) and the assembly constraints by helping in the choice of the assembly sequence.

A new software has been developed by A. Robert named ORASSE, which applies this methodology. In the ORASSE Knowledge version, the user will be guided, step by step, to obtain an optimized design sequence. Its most convenient aspect is the time spared (almost 60\%) in the product structure.

Over recent years, Design Structure Matrix (DSM) was used in different domains, including product design, and led to the emergence of different types of DSMs. [BROWNING 2001] [5] proposes a classification of DSMs into two categories : time-based DSMs and static DSMs. From these categories, four main applications represent the research focus of the use of DSM techniques :

o Component-Based or Architecture DSM : Used for modelling interactions among product components or subsystems. It can be useful for appropriate modularisation of complex systems. Matrix-components DSM which was originally proposed by [KUSIAK] [6].

o Team-Based or Organization DSM : Used for modelling and depicting interactions among organization structures such as design teams.

o Activity-Based or Schedule DSM : Used for modelling processes and activity networks based on information dependencies among design activities.

o Parameter-Based (or Low-Level Schedule) DSM : Used for modelling relationships between design decisions and parameters.

One weakness of these three most common methods for identifying modules is the problem in designing multiple products [HOLTTA-OTTO 2005] [7]. These methods optimize each product of a family but not the family as a whole. To solve this problem, the family can be reduced to a generic product that is used in order to create product variants in a same family thanks to a set of geometric parameters. These geometric parameters can change the dimensions of each variant without any modification of the product architecture. Then, there is a family of products based on the same architecture that can differ from their dimensions. Orasse positioning is then to define a functional architecture from a functional 
analysis combined with the use of a DSM method in order to assign components in each module. Then, independent functional modules can be obtained.

\section{Management of the Parameters Step by Step}

After the internal and external functional analysis is done in ACSP, the user enters the functional parameters in ORASSE Knowledge, because it will be this software, which will simplify the parameters matrix.

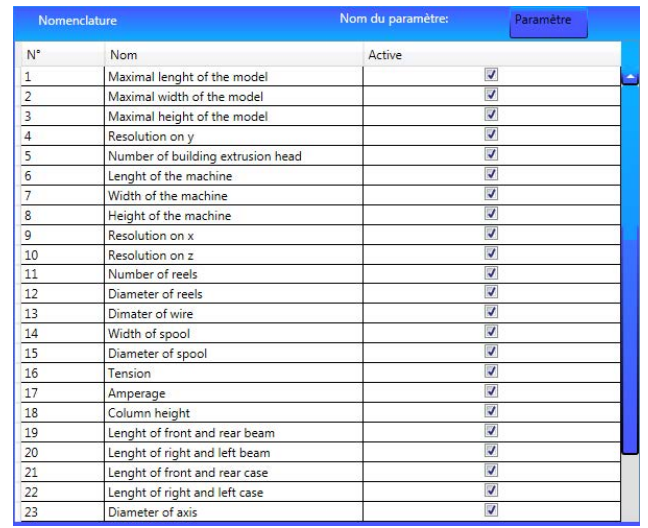

Fig. 2. List of parameters in ORASSE Knowledge

Some pictures can be added, in order to clearly identify the specific parameters with their associated numbers on the component picture.

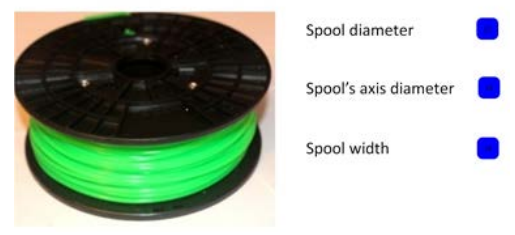

Fig. 3. The plastic coil with its specific parameters

Then the user inserts the list of specific parameters and of functional parameters, associated to each component and to each function, in ORASSE Knowledge, by putting them right under their respective component and their respective function.

In ORASSE Knowledge, the functional parameters and the specific parameters are gathered per colour, depending on which function or component they belong to, and nserted in a matrix, always with their affiliations colour. In this matrix, the links between the different parameters are established. 


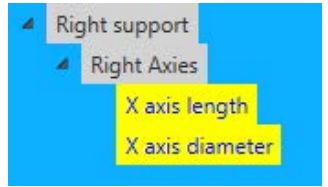

Fig. 4. Components and specific parameters

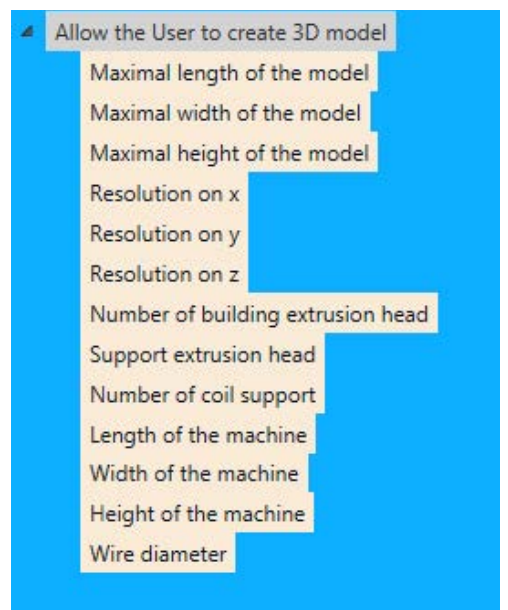

Fig. 5. Functions and functional parameters

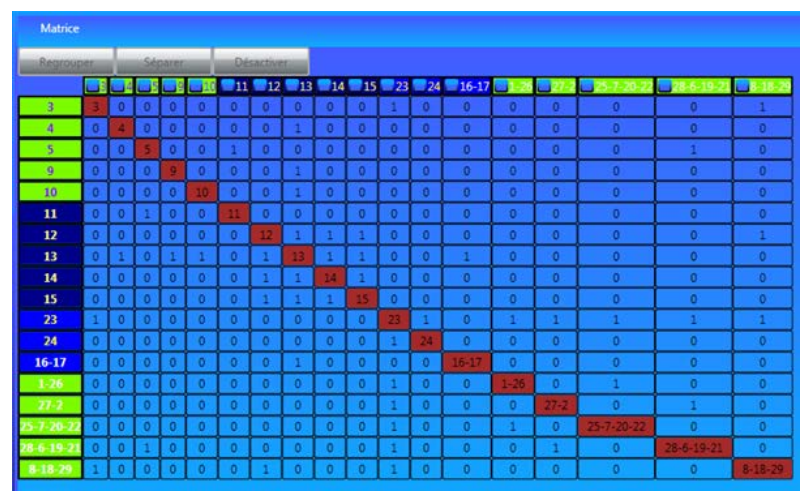

Fig. 6. Matrix parameters-parameters

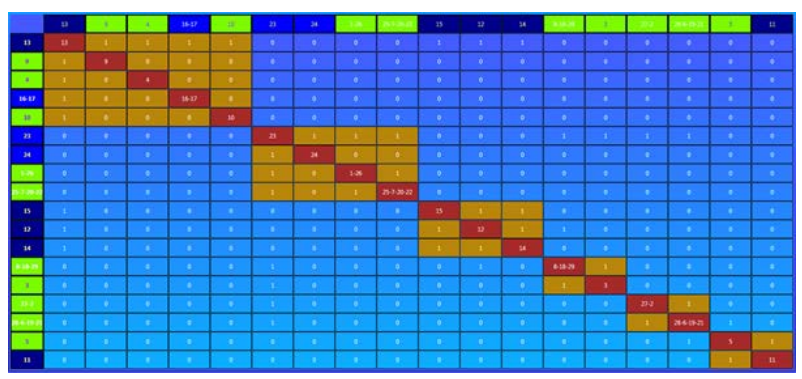

Fig. 7. Matrix parameters-parameters with modules calculated by ORASSE Software 
ORASSE Knowledge automatically generates a graph with the components' numbers. The user has to add the direction of the arrows, which represents the contacts between the various parts, corresponding to the kinematic links between the components. In our example of the 3D Printer, the product design gathers around 300 parameters and some of them are "strongly" linked, as we can see on the graph. ORASSE Knowledge will then simplify the graph by erasing the "strong" contacts and gathering the concerned components.

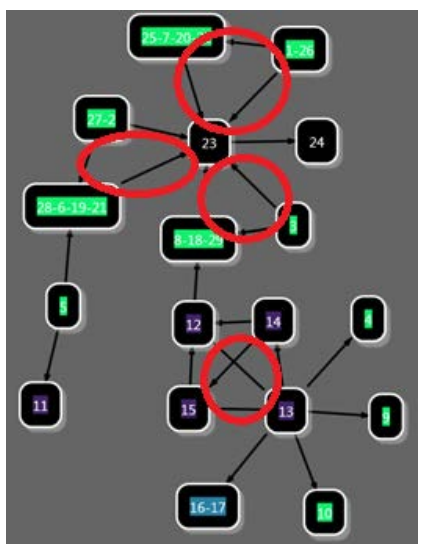

Fig. 8. Graph before simplication

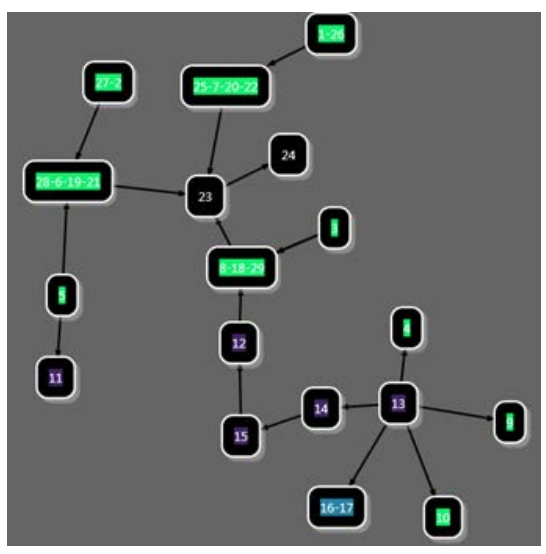

Fig. 9. Simplified Graph

After the graph simplification, the software suggests to start the design sequence with one of the less attached parameters. To continue the design sequence, only the numbers connected to the previous one on the graph are suggested : the user chooses a number that seems plausible for him with his critical design engineering knowledge. At the end, an optimized design sequence is obtained.

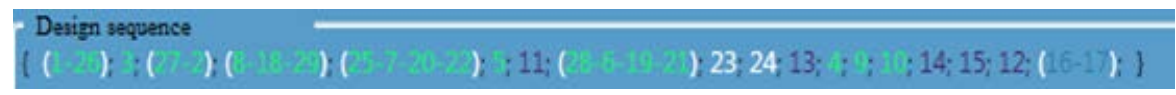

Fig. 10. One of the possible design sequence

These sequence is the most suitable for the product under consideration in this case study and according to the knowledge of the assembly planner. The assembly planner is guided during his/her choice by the decision support system. Using the algorithm, this system informs the assembly planner of three main constraints :

o The functional architecture to respect modular structure of the product by a link of colours 
o The product structure with blocks of components defined with the DSM matrix

o The assembly constraints with relationships between components and assembly planner knowledge

The decision support system guides the assembly planner by proposing choices ranked in accordance with these constraints for selecting the most suitable assembly sequence. But, at any time, he/she can also choose another more suitable solution according to his/her knowledge of the assembly process of the particular company.

These parameters are then inserted in Kadviser by the user. In this software, the parameters are related among themselves by calculus formulation, in such a way that when the parameters are informed in the interfaces, all the functional parameters and the specific parameters will be automatically calculated according to the relation formula in Kadviser. For instance, if the diameter of the ball crew is informed by the user, Kadviser will be able to give its diameter, its length and the screw thread. By this way, the 300 parameters, that the user has to insert, can be reduced to only a dozen, which will control the modularity of the model and then enable the generation of an entire range.

\section{Conclusions}

In this paper, a new approach for the parameters management during a product design has been introduced. It consisted in the generation of a design sequence after having simplified the matrix parameters-parameters with ORASSE Knowledge. The graph being reduced, consequently the parameters are also become less numerous.

To improve their management, the other aspect exploited is the relation between them : in Kadviser, the user can establish calculus formulas to link a parameter with the other ones. This dependence criterion makes the determination of the parameters quicker by inserting some key values, instead of having to recalculate the entire specific parameters : it is the base of the modularity of the product, which will enable the creation of a whole product range.

The use of technological means facilitates the teamwork during a product design, but especially, it helps sparing time. This last point is the most important asset, especially nowadays with the constant competition in the engineering domain : the product designer team will have more time for innovation and the costs will be reduced.

Acknowledgements. The authors would like to thank Samuel Gomes, Frédéric Demoly of the Université de Technologie de Belfort-Montbéliard UTBM (France), for their valuable suggestions on the proposed approach described in the paper, and Julien Bernillon, David Léger students at UTBM for their contributions. 


\section{References}

1. Robert, A. : FARD Model, a new approach to high productive routine design process of modular product using DFA constraints (2011)

2. Toussaint, L. : PLM-based Approach for Design Verification and Validation using Manufacturing Process Knowledge (2010)

3. Petit, C. : Development of a knowledge based engineering application in connection with the PLM and CAD systems

4. Robert, A. : Vers une méthodologie de structuration de la dynamique des interactions au sein du modèle de conception Multi-Domaines et Multi-Vues - Application à la conception de familles de produits modulaires

5. Browning, T.R. : Applying the design structure matrix to system decomposition and integration problems : a review and new directions (2001)

6. Kusiak, A. : Engineering Design : Products, Processes, and Systems. Academic Press (1999)

7. Holtta-Otto, K. : Modular product platform design (2005) 